\title{
Perioperative Bridging Anticoagulation in Patients with Atrial Fibrillation
}

\author{
James D. Douketis, M.D., Alex C. Spyropoulos, M.D., Scott Kaatz, D.O., Richard C. Becker, \\ M.D., Joseph A. Caprini, M.D., Andrew S. Dunn, M.D., David A. Garcia, M.D., Alan Jacobson, \\ M.D., Amir K. Jaffer, M.D., M.B.A., David F. Kong, M.D., Sam Schulman, M.D., Ph.D., \\ Alexander G.G. Turpie, M.B., Vic Hasselblad, Ph.D., and Thomas L. Ortel, M.D., Ph.D. for the \\ BRIDGE Investigators* \\ St. Joseph's Healthcare Hamilton (J.D.D.) and the Department of Medicine (J.D.D.) and Hamilton \\ Health Science Center (S.S., A.G.G.T.), McMaster University, Hamilton, ON, Canada; Hofstra \\ North Shore-Long Island Jewish School of Medicine, Manhasset (A.C.S.), and Mount Sinai \\ Medical Center, New York (A.S.D.) - both in New York; Hurley Medical Center, Flint, MI (S.K.); \\ University of Cincinnati College of Medicine, Cincinnati (R.C.B.); NorthShore University \\ HealthSystem, Evanston (J.A.C.), and Rush University Medical Center, Chicago (A.K.J.) - both \\ in Illinois; University of Washington Medical Center, Seattle (D.A.G.); Veterans Affairs Loma Linda \\ Healthcare System, Loma Linda, CA (A.J.); and Duke Clinical Research Institute (D.F.K., V.H.) \\ and Department of Medicine (T.L.O.), Duke University Medical Center, Durham, NC
}

\section{Abstract \\ BACKGROUND-It is uncertain whether bridging anticoagulation is necessary for patients with atrial fibrillation who need an interruption in warfarin treatment for an elective operation or other elective invasive procedure. We hypothesized that forgoing bridging anticoagulation would be noninferior to bridging with low-molecular-weight heparin for the prevention of perioperative arterial thromboembolism and would be superior to bridging with respect to major bleeding.}

METHODS-We performed a randomized, double-blind, placebo-controlled trial in which, after perioperative interruption of warfarin therapy, patients were randomly assigned to receive bridging anticoagulation therapy with low-molecular-weight heparin (100 IU of dalteparin per kilogram of body weight) or matching placebo administered subcutaneously twice daily, from 3 days before the procedure until 24 hours before the procedure and then for 5 to 10 days after the procedure. Warfarin treatment was stopped 5 days before the procedure and was resumed within 24 hours after the procedure. Follow-up of patients continued for 30 days after the procedure. The primary outcomes were arterial thromboembolism (stroke, systemic embolism, or transient ischemic attack) and major bleeding.

\footnotetext{
Address reprint requests to Dr. Ortel at Duke University Medical Center, Box 3422, Durham, NC, 27710, or at thomas.ortel@duke.edu.

A complete list of investigators in the Bridging Anticoagulation in Patients who Require Temporary Interruption of Warfarin Therapy for an Elective Invasive Procedure or Surgery (BRIDGE) study is provided in the Supplementary Appendix, available at NEJM.org. No other potential conflict of interest relevant to this article was reported.

Disclosure forms provided by the authors are available with the full text of this article at NEJM.org.
} 
RESULTS-In total, 1884 patients were enrolled, with 950 assigned to receive no bridging therapy and 934 assigned to receive bridging therapy. The incidence of arterial thromboembolism was $0.4 \%$ in the no-bridging group and $0.3 \%$ in the bridging group (risk difference, 0.1 percentage points; $95 \%$ confidence interval [CI], -0.6 to $0.8 ; \mathrm{P}=0.01$ for noninferiority). The incidence of major bleeding was $1.3 \%$ in the no-bridging group and $3.2 \%$ in the bridging group (relative risk, $0.41 ; 95 \% \mathrm{CI}, 0.20$ to $0.78 ; \mathrm{P}=0.005$ for superiority).

CONCLUSIONS-In patients with atrial fibrillation who had warfarin treatment interrupted for an elective operation or other elective invasive procedure, forgoing bridging anticoagulation was noninferior to perioperative bridging with low-molecular-weight heparin for the prevention of arterial thromboembolism and decreased the risk of major bleeding. (Funded by the National Heart, Lung, and Blood Institute of the National Institutes of Health; BRIDGE ClinicalTrials.gov number, NCT00786474.)

For patients with atrial fibrillation who are receiving warfarin and require an elective operation or other elective invasive procedure, the need for bridging anticoagulation during perioperative interruption of warfarin treatment has long been uncertain. ${ }^{1-3}$ Each year, this common clinical scenario affects approximately one in six warfarin-treated patients with atrial fibrillation. ${ }^{4,5}$ Warfarin treatment is typically stopped 5 days before an elective procedure to allow its anticoagulant effect to wane; it is resumed after the procedure, when hemostasis is secured, at which point 5 to 10 days of treatment is required to attain therapeutic anticoagulation. ${ }^{6,7}$ During the interruption of warfarin treatment, bridging anticoagulation therapy, typically with low-molecular-weight heparin, can be given to minimize the time that patients do not have an adequate level of anticoagulation, with the intent of minimizing the risk of perioperative arterial thromboembolism, such as stroke. ${ }^{6}$

Multiple observational studies have assessed the timing and dosing of perioperative bridging with low-molecular-weight heparin. ${ }^{8-15}$ However, the fundamental question of whether bridging anticoagulation is necessary during perioperative warfarin interruption has remained unanswered. ${ }^{16-18}$ Because of the lack of evidence, practice guidelines have provided weak and inconsistent recommendations regarding the need for bridging anticoagulation. ${ }^{19-21}$

Against this background, the Bridging Anticoagulation in Patients who Require Temporary Interruption of Warfarin Therapy for an Elective Invasive Procedure or Surgery (BRIDGE) trial was designed to address a simple question: in patients with atrial fibrillation, is heparin bridging needed during interruption of warfarin therapy before and after an operation or other invasive procedure? We hypothesized that forgoing bridging altogether would be noninferior to bridging with low-molecular-weight heparin for the prevention of perioperative arterial thromboembolism and would be superior to bridging with regard to the outcome of major bleeding.

\section{METHODS}

\section{STUDY DESIGN AND OVERSIGHT}

The BRIDGE trial was a randomized, double-blind, placebo-controlled trial. The protocol (available with the full text of this article at NEJM.org) was designed by the steering 
committee (see the Supplementary Appendix, available at NEJM.org, for a full list of trial personnel) and approved by the institutional review board at each participating clinical center. The Duke Clinical Research Institute managed the study. The clinical coordinating center was responsible for study coordination, randomization, and distribution of the study drug. The data coordinating center was responsible for maintenance of the study database, data validation, and analyses. Eisai donated the dalteparin, and University of Iowa Pharmaceuticals prepared the matching placebo. Eisai had no role in the design or conduct of the study, the analysis of the data, or the preparation of the manuscript. The steering committee vouches for the completeness and accuracy of the data and analyses and for the fidelity of this report to the trial protocol.

\section{PATIENTS}

Patients were eligible to participate in the trial if they were 18 years of age or older; had chronic (permanent or paroxysmal) atrial fibrillation or flutter, confirmed by means of previous electrocardiography or pacemaker interrogation (patients with atrial fibrillation associated with valvular disease, including mitral valve disease, were eligible); had received warfarin therapy for 3 months or longer, with an international normalized ratio (INR) therapeutic range of 2.0 to 3.0; were undergoing an elective operation or other elective invasive procedure that required interruption of warfarin therapy; and had at least one of the following $\mathrm{CHADS}_{2}$ stroke risk factors: congestive heart failure or left ventricular dysfunction, hypertension, age of 75 years or older, diabetes mellitus, or previous ischemic stroke, systemic embolism, or transient ischemic attack. Patients were not eligible if they had one or more of the following: a mechanical heart valve; stroke, systemic embolism, or transient ischemic attack within the previous 12 weeks; major bleeding within the previous 6 weeks; creatinine clearance of less than $30 \mathrm{ml}$ per minute; platelet count of less than $100 \times 10^{3}$ per cubic millimeter; or planned cardiac, intracranial, or intraspinal surgery. A complete list of the trial inclusion and exclusion criteria is provided in the Supplementary Appendix. All participants provided written informed consent.

\section{PROCEDURES}

Patients were randomly assigned to receive bridging anticoagulation therapy with dalteparin sodium (100 IU per kilogram of body weight administered subcutaneously twice daily) or to receive no bridging therapy (i.e., a matching subcutaneous placebo) from 3 days before the procedure until 24 hours before the procedure and then for 5 to 10 days after the procedure. Randomization was stratified according to study center either with the use of an interactive voice-response system with a toll-free telephone number and access codes or through the Internet. The study drugs were provided in identical vials.

The administration of study drug followed a standardized perioperative management protocol (Fig. 1). Warfarin treatment was stopped 5 days before the procedure, and administration of the study drug (dalteparin or matching placebo) was started 3 days before the procedure. The last preprocedure dose of dalteparin or placebo was given in the morning approximately 24 hours before the procedure. ${ }^{22,23}$ Warfarin treatment was restarted on the evening of or the day after the procedure, at the patient's usual dose. Administration of dalteparin or placebo was resumed 12 to 24 hours after a minor (or low-bleeding-risk) 
procedure and 48 to 72 hours after a major (or high-bleeding-risk) procedure. ${ }^{8,10}$ The designation of a procedure as having a low or high bleeding risk was guided by means of a classification scheme (see Table S1 in the Supplementary Appendix), but the final determination of risk was left to the investigator's discretion. The patient continued to take the study drug after the procedure until the INR was 2 or higher on one occasion. Patients had follow-up encounters by telephone weekly, with the final encounter 30 to 37 days after the procedure. Perioperative management of antiplatelet therapy was left to the site investigator's discretion.

\section{STUDY OUTCOMES}

All study outcomes were assessed by 37 days after the procedure. The primary efficacy outcome was arterial thromboembolism, including stroke (ischemic or hemorrhagic), transient ischemic attack, and systemic embolism, and the primary safety outcome was major bleeding. The secondary efficacy outcomes were acute myocardial infarction, deepvein thrombosis, pulmonary embolism, and death, and the secondary safety outcome was minor bleeding. The definitions of the outcomes are provided in the Supplementary Appendix. All study outcomes were independently and blindly adjudicated.

\section{STATISTICAL ANALYSIS}

The primary efficacy outcome was arterial thromboembolism at 30 days. The initial samplesize estimates for arterial thromboembolism were based on the results of contemporaneous cohort studies, which suggested that the rate in the bridging group would be $1.0 \%{ }^{8-10,24,25}$ We also assumed that the rate in the no-bridging group would be $1.0 \%$. The primary analysis of efficacy was a noninferiority analysis with a one-sided test at the 0.025 level. The noninferiority margin was set at $1.0 \%$. We determined that the hypothesis of inferiority would be rejected if the upper boundary of the $95 \%$ confidence interval for the difference in rates would be less than 1.0 percentage point. We prespecified that the $95 \%$ confidence interval for the difference in event rates would be calculated with the use of methods based on Barnard's test, ${ }^{26}$ because this test permits the calculation of confidence intervals in analyses with small sample sizes. The confidence interval values were calculated with the use of StatXact software, version 9 (Cytel). ${ }^{27}$

The primary safety outcome was major bleeding at 30 days after the procedure. The null hypothesis of no difference in the incidence of major bleeding was tested with a two-sided test at the 0.05 level. The expected bleeding rates were $1.0 \%$ in the no-bridging arm and $3.0 \%$ in the bridging arm. The $\mathrm{P}$ value was calculated with the use of Fisher's mid-P test, as implemented in SAS software, version 9.3 (SAS Institute), and the 95\% confidence interval was a likelihood-ratio confidence interval calculated with the same version of SAS.

We calculated that a sample of 1641 patients per group would give the study $80 \%$ power to detect the noninferiority of no bridging therapy, assuming a rate of arterial thromboembolism of $1.0 \%$ in each group and a noninferiority margin of $1.0 \%$, at a one-sided alpha level of 0.025 for arterial thromboembolism and a two-sided alpha level of 0.05 for bleeding. With a $10 \%$ allowance for patients withdrawing from the study, the required 
sample size was 1813 per group. We calculated that this sample size would also give the study more than $99 \%$ power to detect the expected difference in bleeding rates.

After approximately 850 patients had been enrolled, it was clear that the rate of arterial thromboembolism, as assessed by investigators who were unaware of the study-group assignments, was less than $0.5 \%$, and we determined that a revised sample size of 2526 would provide at least $90 \%$ power for each primary end point. After 1720 patients were enrolled, the rate of arterial thromboembolism was $0.46 \%$, and the bleeding rate was $2.3 \%$ in the entire population. A revised sample size of 1882 was calculated on the basis of the estimate that this would provide nearly $90 \%$ power for the two primary end points.

\section{RESULTS}

PATIENTS

As shown in Figure 2, we recruited 1884 patients during the period from July 2009 through December 2014 at 108 sites in the United States and Canada; 950 patients were assigned to the placebo (no-bridging) group, and 934 patients were assigned to receive bridging treatment with dalteparin (bridging group). Table 1 shows the characteristics of the patients at baseline. The mean age of the patients was 71.7 years, and $73.4 \%$ of patients were male; the mean body weight was $95.8 \mathrm{~kg}$. The mean $\mathrm{CHADS}_{2}$ score $\left(\mathrm{CHADS}_{2}\right.$ scores range from 1 to 6 , with higher scores indicating a greater risk of stroke) was $2.3 ; 38.3 \%$ of patients had a $\mathrm{CHADS}_{2}$ score of 3 or higher. A total of $34.7 \%$ of the patients were taking aspirin, and $7.2 \%$ were taking another antiplatelet drug.

Of the 1884 patients enrolled in the trial, 1722 actually underwent the anticipated procedure (as-treated group), and 162 did not. The categories and types of operations and procedures that the participants underwent are shown in Table S2 in the Supplementary Appendix. The most common procedures were gastrointestinal (44.0\%), cardiothoracic (17.2\%), and orthopedic $(9.2 \%)$. Overall, $89.4 \%$ of patients underwent a procedure that was classified as minor (low bleeding risk) according to the prespecified classification; however, $69.1 \%$ were treated as having a low bleeding risk by the site investigator.

\section{PERIOPERATIVE ANTICOAGULANT MANAGEMENT}

The mean $( \pm \mathrm{SD})$ number of doses of study drug administered was $5.0 \pm 1.1$ before the procedure and 16.0 \pm 7.9 after the procedure (Table 2). The mean dose of dalteparin administered was $9093 \pm 2240$ IU subcutaneously twice daily. Adherence to the study-drug protocol, defined as administration of $100 \%$ of protocol-specified doses of study drug, was $86.5 \%$ before the procedure and $96.5 \%$ after the procedure.

\section{STUDY OUTCOMES}

Of the 1884 patients enrolled in the trial, 71 discontinued participation and did not provide outcome data; therefore, data from 1813 patients were available for the analysis (Fig. 2). At 30 days after the procedure, the incidence of arterial thromboembolism was $0.4 \%$ (four events among 918 patients) in the no-bridging group and $0.3 \%$ (three events among 895 patients) in the bridging group (mean between-group difference, 0.1 percentage points; $95 \%$ 
confidence interval $[\mathrm{CI}],-0.6$ to $0.8 ; \mathrm{P}=0.01$ for noninferiority; $\mathrm{P}=0.73$ for superiority) (Table 3). In an as-treated analysis, the rates of arterial thromboembolism were $0.3 \%$ (three events among 875 patients) in the no-bridging group and $0.4 \%$ (three events among 847 patients) in the bridging group (mean between-group difference, 0.0 percentage points; $95 \%$ CI, -0.7 to $0.7 ; \mathrm{P}=0.006$ for noninferiority). Patients in whom arterial thromboembolism occurred had a mean CHADS $_{2}$ score of 2.6 (range, 1 to 4), and five of the seven events occurred after a minor procedure. The median time to an arterial thromboembolism event after the procedure was 19.0 days (interquartile range, 6.0 to 23.0).

Major bleeding occurred in 1.3\% of the patients (12 of 918) in the no-bridging group and in $3.2 \%$ ( 29 of 895 ) in the bridging group, which indicated that no bridging was superior to bridging with regard to major bleeding (relative risk, $0.41 ; 95 \% \mathrm{CI}, 0.20$ to $0.78 ; \mathrm{P}=0.005$ ). None of the instances of major bleeding were fatal. Forgoing bridging was associated with a risk of minor bleeding that was significantly lower than the risk associated with bridging $(12.0 \%$ vs. $20.9 \%, \mathrm{P}<0.001)$. The median time to a major bleeding outcome after the procedure was 7.0 days (interquartile range, 4.0 to 18.0 ).

There was no significant difference between the groups in the rates of acute myocardial infarction, deep-vein thrombosis, pulmonary embolism, or death. Information on the causes of death and times to death is provided in Table S3 in the Supplementary Appendix.

\section{DISCUSSION}

We found that in patients with atrial fibrillation who require perioperative interruption of warfarin treatment for an elective procedure, a strategy of discontinuing warfarin treatment without the use of bridging anticoagulation was noninferior to the use of bridging anticoagulation for the prevention of arterial thromboembolism; in addition, bridging conferred a risk of major bleeding that was nearly triple the risk associated with no bridging. There was also less minor bleeding without bridging than there was with bridging, and there was no significant difference between the groups with regard to myocardial infarction, venous thromboembolism, or death. Taken together, these findings show that there is a net clinical benefit in favor of a strategy of forgoing bridging, as compared with perioperative bridging with low-molecular-weight heparin.

The findings in our trial are consistent with those from nonrandomized comparisons of these strategies. A meta-analysis of observational studies involving a total of 12,278 patients with atrial fibrillation or mechanical heart valves who received or did not receive bridging with low-molecular-weight heparin showed no significant difference in the rate of arterial thromboembolism (odds ratio with bridging, $0.80 ; 95 \% \mathrm{CI}, 0.42$ to 1.54 ) but a higher rate of major bleeding (odds ratio, 3.60; 95\% CI, 1.52 to 8.50 ) in association with bridging. ${ }^{28}$ In a substudy of the Randomized Evaluation of Long-Term Anticoagulation Therapy (RE-LY), ${ }^{29}$ in which patients with atrial fibrillation were randomly assigned to receive warfarin or dabigatran in an open-label manner, bridging anticoagulation was associated with a rate of major bleeding that was higher than that associated with no bridging $(6.8 \%$ vs. $1.6 \%$, $\mathrm{P}<0.001)$ among 1424 warfarin-treated patients who had treatment interruption for an elective procedure, and there was no significant effect on arterial thromboembolism $(0.5 \%$ 
vs. $0.2 \%, \mathrm{P}=0.32) .{ }^{30}$ The Outcomes Registry for Better Informed Treatment of Atrial Fibrillation study (ORBIT-AF), which involved 2200 patients with atrial fibrillation who required an elective procedure, also showed a higher rate of bleeding if bridging anticoagulation therapy was used during perioperative interruption of warfarin treatment. ${ }^{31}$

The rationale for the use of bridging anticoagulation therapy has been anchored on the premise that the associated higher bleeding risk was clinically acceptable because it would be offset by a lower risk of perioperative arterial throm-boembolism. ${ }^{32}$ The findings from the BRIDGE trial as well as from nonrandomized studies suggest that the perioperative risk of arterial thromboembolism in patients with atrial fibrillation during interruption of warfarin treatment may have been overstated and may not be mitigated by bridging anticoagulation. Indeed, the mechanisms of perioperative arterial thromboembolism may be more closely related to factors such as the type of procedure ${ }^{33}$ and to intraoperative alterations in blood pressure. ${ }^{34}$ The premise that warfarin interruption leads to rebound hypercoagulability and that the milieu of the procedure confers a prothrombotic state, which in turn leads to arterial thromboembolism, is not supported by the results of this trial. ${ }^{35-37}$

There are potential limitations of the BRIDGE trial. First, although we aimed to recruit a representative sample of patients with atrial fibrillation for whom bridging anticoagulation is normally considered, certain groups were underrepresented. Few patients had a $\mathrm{CHADS}_{2}$ score of 5 or 6 , although the mean score of 2.3 is similar to that among patients with atrial fibrillation who were assessed in recent trials and patient registries, in which the mean scores were between 2.1 and 2.8. ${ }^{29,38-40}$ Patients undergoing major surgical procedures associated with high rates of arterial thromboembolism and bleeding (e.g., carotid endarterectomy, major cancer surgery, cardiac surgery, or neurosurgery $)^{19,33}$ were not represented in the trial, although the procedures performed were representative of the most common interventions patients undergo during an interruption of therapeutic anticoagulation, the majority of which are low-risk procedures, such as colonoscopy or ambulatory surgery. ${ }^{4,5,41}$ In addition, the findings should not be applied to patients with mechanical heart valves, who were specifically not included in the trial.

Second, the overall rate of arterial thromboembolism was lower than expected, which potentially affected the power of the trial to detect a benefit associated with bridging. Although we had expected perioperative arterial thromboembolism rates to be approximately $1.0 \%, 8,9,12,24$ the observed rate $(0.4 \%)$ is similar to rates in recent studies involving patients who had perioperative interruption of warfarin treatment. ${ }^{4,5,31,42}$ In addition, the noninferiority margin we selected turned out to be large in relation to the actual observed event rate; it reflected the original estimate of the event rate as specified in the trial protocol.

Third, the observed rate of major bleeding in the bridging group (3.2\%, with no instances of fatal bleeding) may be considered to be modest. However, our bridging protocol was designed to minimize bleeding, and the higher rates of bleeding reported in other studies of bridging anticoagulation probably reflect resumption of bridging therapy too soon after operations with a high bleeding risk ${ }^{10,43}$ or a lack of standardized bridging protocols..$^{28,30}$ 
Fourth, the reduction in the study sample size may raise concerns. This reduction was driven by the lower rate of arterial thromboembolism overall, with the proviso that power was maintained to address the primary study hypotheses. Although extending the trial was considered, this was not done because the added statistical power would have been negligible and because recruitment had been challenging throughout the course of the trial.

Finally, one may contend that the trial findings have diminished relevance because of the decreasing use of warfarin in the treatment of patients with atrial fibrillation, given the availability of the newer direct oral anticoagulants. ${ }^{6}$ However, warfarin remains widely used among patients with atrial fibrillation. ${ }^{44-46}$ Furthermore, the trial findings may also apply to the newer agents. In the substudy of the RE-LY trial discussed above, dabigatran-treated patients who had treatment interruption for an elective procedure had more major bleeding with bridging therapy than without bridging therapy, and there was no significant effect on arterial thromboembolism. ${ }^{30}$

In conclusion, in the BRIDGE trial, we found that for patients with atrial fibrillation who require temporary interruption of warfarin treatment for an elective operation or other elective invasive procedure, a strategy of forgoing bridging anticoagulation was noninferior to perioperative bridging with low-molecular-weight heparin for the prevention of arterial thromboembolism. The strategy of forgoing bridging treatment also decreased the risk of major bleeding.

\title{
Supplementary Material
}

Refer to Web version on PubMed Central for supplementary material.

\section{Acknowledgments}

\begin{abstract}
Supported by grants from the National Heart, Lung, and Blood Institute for the clinical coordinating center (U01HL087229, to Dr. Ortel) and for the data coordinating center (U01HL086755, to Dr. Hasselblad). Eisai supplied the active drug, dalteparin sodium (Fragmin), through an unrestricted investigator-initiated grant to Dr. Ortel.
\end{abstract}

Dr. Douketis reports receiving fees for serving on advisory boards from Biotie, Portola, and the Medicines Company, honoraria from Bristol-Myers Squibb, Pfizer, and Sanofi Aventis, consulting fees from Boehringer Ingelheim, Bayer, Janssen, Bristol-Myers Squibb, Daiichi-Sankyo, and Actelion, and grant support from Boehringer Ingelheim; Dr. Spyropoulos, receiving consulting fees from Janssen, Boehringer Ingelheim, Daiichi-Sankyo, and Pfizer, and grant support from Daiichi-Sankyo; Dr. Kaatz, receiving consulting and lecture fees from Boehringer Ingelheim, Bristol-Myers Squibb/Pfizer Alliance, Janssen, CSL Behring, and Daiichi-Sankyo, and grant support through his institution from Boehringer Ingelheim, Bristol-Myers Squibb/ Pfizer Alliance, Janssen, Iverson Genetics Diagnostics/Medicare, and Blue Cross Blue Shield of Michigan; Dr. Becker, receiving fees for serving on scientific advisory boards from Janssen, Portola, and Daiichi-Sankyo; Dr. Caprini, receiving fees for serving on advisory boards from Bristol-Myers Squibb and Pfizer, fees for serving on a steering committee from Janssen, and lecture fees from Sanofi Aventis; Dr. Garcia, receiving consulting fees from Pfizer, Genzyme, Boehringer Ingelheim, Bristol-Myers Squibb, Portola, and Daiichi-Sankyo, and grant support from Bayer; Dr. Jacobson, receiving consulting fees from Bristol-Myers Squibb/Pfizer Alliance, Daiichi-Sankyo, Boehringer Ingelheim, Janssen, Roche Diagnostics, and Alere, and lecture fees from Bristol-Myers Squibb/Pfizer Alliance, DaiichiSankyo, Boehringer Ingelheim, and Janssen; Dr. Jaffer, receiving consulting fees from Pfizer, Janssen, DaiichiSankyo, Boehringer Ingelheim, Marathon, and Medtronic; and Dr. Ortel, receiving consulting fees from Instrumentation Laboratory, CSL Behring, and Daiichi-Sankyo, and grant support from Instrumentation Laboratory.

We thank Andrei L. Kindzelski, who served as the National Institutes of Health program official; Jill Lynch, University of Iowa Pharmaceuticals, who was the manufacturer of the matching placebo; and James Bernstein, Live Oak Pharmaceuticals Consulting, for pharmacy consultancy regarding the processes involved in the manufacture of the matching placebo and for packaging the study drug kits for distribution to the trial sites. 


\section{References}

1. Kearon C, Hirsh J. Management of anticoagulation before and after elective surgery. N Engl J Med. 1997; 336:1506-11. [PubMed: 9154771]

2. Piazza G, Goldhaber SZ. Periprocedural management of the chronically anticoagulated patient: critical pathways for bridging therapy. Crit Pathw Cardiol. 2003; 2:96-103. [PubMed: 18340326]

3. Gallego P, Apostolakis S, Lip GY. Bridging evidence-based practice and practice-based evidence in periprocedural anticoagulation. Circulation. 2012; 126:1573-6. [PubMed: 23008469]

4. Healey JS, Eikelboom J, Douketis J, et al. Periprocedural bleeding and thromboembolic events with dabigatran compared with warfarin: results from the Randomized Evaluation of Long-Term Anticoagulation Therapy (RE-LY) randomized trial. Circulation. 2012; 126:343-8. Erratum, Circulation 2012;126(10):e160. [PubMed: 22700854]

5. Garcia D, Alexander JH, Wallentin L, et al. Management and clinical outcomes in patients treated with apixaban vs warfarin undergoing procedures. Blood. 2014; 124:3692-8. [PubMed: 25320240]

6. Baron TH, Kamath PS, McBane RD. Management of antithrombotic therapy in patients undergoing invasive procedures. N Engl J Med. 2013; 368:2113-24. [PubMed: 23718166]

7. Schulman S, Hwang HG, Eikelboom JW, Kearon C, Pai M, Delaney J. Loading dose vs. maintenance dose of warfarin for reinitiation after invasive procedures: a randomized trial. $\mathrm{J}$ Thromb Haemost. 2014; 12:1254-9. [PubMed: 24837794]

8. Douketis JD, Johnson JA, Turpie AG. Low-molecular-weight heparin as bridging anticoagulation during interruption of warfarin: assessment of a standardized periprocedural anticoagulation regimen. Arch Intern Med. 2004; 164:1319-26. [PubMed: 15226166]

9. Kovacs MJ, Kearon C, Rodger M, et al. Single-arm study of bridging therapy with low-molecularweight heparin for patients at risk of arterial embolism who require temporary interruption of warfarin. Circulation. 2004; 110:1658-63. [PubMed: 15364803]

10. Dunn AS, Spyropoulos AC, Turpie AG. Bridging therapy in patients on long-term oral anticoagulants who require surgery: the Prospective Peri-operative Enoxaparin Cohort Trial (PROSPECT). J Thromb Haemost. 2007; 5:2211-8. [PubMed: 17697140]

11. Halbritter KM, Wawer A, Beyer J, Oettler W, Schellong SM. Bridging anticoagulation for patients on long-term vitamin-K-antagonists: a prospective 1 year registry of 311 episodes. J Thromb Haemost. 2005; 3:2823-5. [PubMed: 16359528]

12. Spyropoulos AC, Turpie AGG, Dunn AS, et al. Clinical outcomes with unfractionated heparin or low-molecular-weight heparin as bridging therapy in patients on long-term oral anticoagulants: the REGIMEN registry. J Thromb Haemost. 2006; 4:1246-52. [PubMed: 16706967]

13. Wysokinski WE, McBane RD, Daniels PR, et al. Periprocedural anticoagulation management of patients with nonvalvular atrial fibrillation. Mayo Clin Proc. 2008; 83:639-45. [PubMed: 18533080]

14. Pengo V, Cucchini U, Denas G, et al. Standardized low-molecular-weight heparin bridging regimen in outpatients on oral anticoagulants undergoing invasive procedure or surgery: an inception cohort management study. Circulation. 2009; 119:2920-7. [PubMed: 19470892]

15. Malato A, Saccullo G, Lo Coco L, et al. Patients requiring interruption of long-term oral anticoagulant therapy: the use of fixed sub-therapeutic doses of low-molecular-weight heparin. J Thromb Haemost. 2010; 8:107-13. [PubMed: 19817996]

16. Ansell JE. The perioperative management of warfarin therapy. Arch Intern Med. 2003; 163:881-3. [PubMed: 12719195]

17. BRIDGE Study Investigators. Bridging anticoagulation: is it needed when warfarin is interrupted around the time of a surgery or procedure? Circulation. 2012; 125(12):e496-e498. [PubMed: 22451610]

18. Patel JP, Arya R. The current status of bridging anticoagulation. Br J Haematol. 2014; 164:619-29. [PubMed: 24224681]

19. Douketis JD, Berger PB, Dunn AS, et al. The perioperative management of antithrombotic therapy: American College of Chest Physicians Evidence-Based Clinical Practice Guidelines (8th Edition). Chest. 2008; 133(Suppl):299S-339S. [PubMed: 18574269] 
20. Fuster V, Rydén LE, Cannom DS, et al. 2011 ACCF/AHA/HRS focused updates incorporated into the ACC/AHA/ESC 2006 Guidelines for the management of patients with atrial fibrillation: a report of the American College of Cardiology Foundation/American Heart Association Task Force on Practice Guidelines developed in partnership with the European Society of Cardiology and in collaboration with the European Heart Rhythm Association and the Heart Rhythm Society. J Am Coll Cardiol. 2011; 57(11):e101-e198. [PubMed: 21392637]

21. Douketis JD, Spyropoulos AC, Spencer FA, et al. Perioperative management of antithrombotic therapy: Antithrombotic Therapy and Prevention of Thrombosis, 9th ed: American College of Chest Physicians Evidence-Based Clinical Practice Guidelines. Chest. 2012; 141(Suppl):e326Se350S. [PubMed: 22315266]

22. O'Donnell MJ, Kearon C, Johnson J, et al. Brief communication: preoperative anticoagulant activity after bridging low-molecular-weight heparin for temporary interruption of warfarin. Ann Intern Med. 2007; 146:184-7. [PubMed: 17283349]

23. Douketis JD, Woods K, Foster GA, Crowther MA. Bridging anticoagulation with low-molecularweight heparin after interruption of warfarin therapy is associated with a residual anticoagulant effect prior to surgery. Thromb Haemost. 2005; 94:528-31. [PubMed: 16268467]

24. Dunn AS, Turpie AG. Perioperative management of patients receiving oral anticoagulants: a systematic review. Arch Intern Med. 2003; 163:901-8. [PubMed: 12719198]

25. Garcia DA, Regan S, Henault LE, et al. Risk of thromboembolism with short-term interruption of warfarin therapy. Arch Intern Med. 2008; 168:63-9. [PubMed: 18195197]

26. Barnard GA. Significance tests for $2 \times 2$ tables. Biometrika. 1947; 34:123-38. [PubMed: 20287826]

27. StatXact Version 9 with Cytel Studio. Cambridge, MA: CYTEL Software; 2010.

28. Siegal D, Yudin J, Kaatz S, Douketis JD, Lim W, Spyropoulos AC. Periprocedural heparin bridging in patients receiving vitamin $\mathrm{K}$ antagonists: systematic review and meta-analysis of bleeding and thromboembolic rates. Circulation. 2012; 126:1630-9. [PubMed: 22912386]

29. Connolly SJ, Ezekowitz MD, Yusuf S, et al. Dabigatran versus warfarin in patients with atrial fibrillation. N Engl J Med. 2009; 361:1139-51. [PubMed: 19717844]

30. Douketis JD, Healey JS, Brueckmann M, et al. Perioperative bridging anticoagulation during dabigatran or warfarin interruption among patients who had an elective surgery or procedure: substudy of the RE-LY trial. Thromb Haemost. 2015; 113:625-32. [PubMed: 25472710]

31. Steinberg BA, Peterson ED, Kim S, et al. Use and outcomes associated with bridging during anticoagulation interruptions in patients with atrial fibrillation: findings from the Outcomes Registry for Better Informed Treatment of Atrial Fibrillation (ORBIT-AF). Circulation. 2015; 131:488-94. [PubMed: 25499873]

32. Spyropoulos AC. Pro: "Bridging anticoagulation is needed during warfarin interruption in patients who require elective surgery. Thromb Haemost. 2012; 108:213-6. [PubMed: 22688503]

33. Kaatz S, Douketis JD, Zhou H, Gage BF, White RH. Risk of stroke after surgery in patients with and without chronic atrial fibrillation. J Thromb Haemost. 2010; 8:884-90. [PubMed: 20096001]

34. Cheung CC, Martyn A, Campbell N, et al. Predictors of intraoperative hypotension and bradycardia. Am J Med. 2015; 128:532-8. [PubMed: 25541033]

35. Grip L, Blombäck M, Schulman S. Hypercoagulable state and thromboembolism following warfarin withdrawal in post-myocardial-infarction patients. Eur Heart J. 1991; 12:1225-33. [PubMed: 1782954]

36. Palareti G, Legnani C. Warfarin withdrawal: pharmacokinetic-pharmacodynamic considerations. Clin Pharmacokinet. 1996; 30:300-13. [PubMed: 8983860]

37. Kosir MA, Schmittinger L, Barno Winarski L, et al. Prospective double-arm study of fibrinolysis in surgical patients. J Surg Res. 1998; 74:96-101. [PubMed: 9536981]

38. Granger CB, Alexander JH, McMurray JJ, et al. Apixaban versus warfarin in patients with atrial fibrillation. N Engl J Med. 2011; 365:981-92. [PubMed: 21870978]

39. Giugliano RP, Ruff CT, Braunwald, et al. Edoxaban versus warfarin in patients with atrial fibrillation. N Engl J Med. 2013; 369:2093-104. [PubMed: 24251359] 
40. Graham DJ, Reichman ME, Wernecke M, et al. Cardiovascular, bleeding, and mortality risks in elderly Medicare patients treated with dabigatran or warfarin for nonvalvular atrial fibrillation. Circulation. 2015; 131:157-64. [PubMed: 25359164]

41. Beyer-Westendorf J, Gelbricht Förster K, et al. Peri-interventional management of novel oral anticoagulants in daily care: results from the prospective -Dresden NOAC registry. Eur Heart J. 2014; 35:1888-96. [PubMed: 24394381]

42. Sherwood MW, Douketis JD, Patel, et al. Outcomes of temporary interruption of rivaroxaban compared with warfarin in patients with nonvalvular atrial fibrillation: results from the Rivaroxaban Once Daily, Oral, Direct Factor Xa Inhibition Compared With Vitamin K Antagonism for Prevention of Stroke and Embolism Trial in Atrial Fibrillation (ROCKET AF). Circulation. 2014; 129:1850-9. [PubMed: 24552831]

43. Birnie DH, Healey JS, Wells GA, et al. Pacemaker or defibrillator surgery without interruption of anticoagulation. N Engl J Med. 2013; 368:2084-93. [PubMed: 23659733]

44. Xu Y, Holbrook AM, Simpson CS, Dowlatshahi D, Johnson AP. Prescribing V, patterns of novel oral anticoagulants following regulatory approval for atrial fibrillation in Ontario, Canada: a population-based descriptive analysis. CMAJ Open. 2013; 1:E115-9.

45. Desai NR, Krumme AA, Schneeweiss S, et al. Patterns of initiation of oral anticoagulants in patients with atrial fibrillation- quality and cost implications. Am J Med. 2014; 127(11):1075.e11082.e1. [PubMed: 24859719]

46. Olesen JB, Sørensen R, Hansen ML, et al. Non-vitamin K antagonist oral anticoagulation agents in anticoagulant naïve atrial fibrillation patients: Danish nationwide descriptive data 2011-2013.

Europace. 2015; 17:187-93. [PubMed: 25236181] 


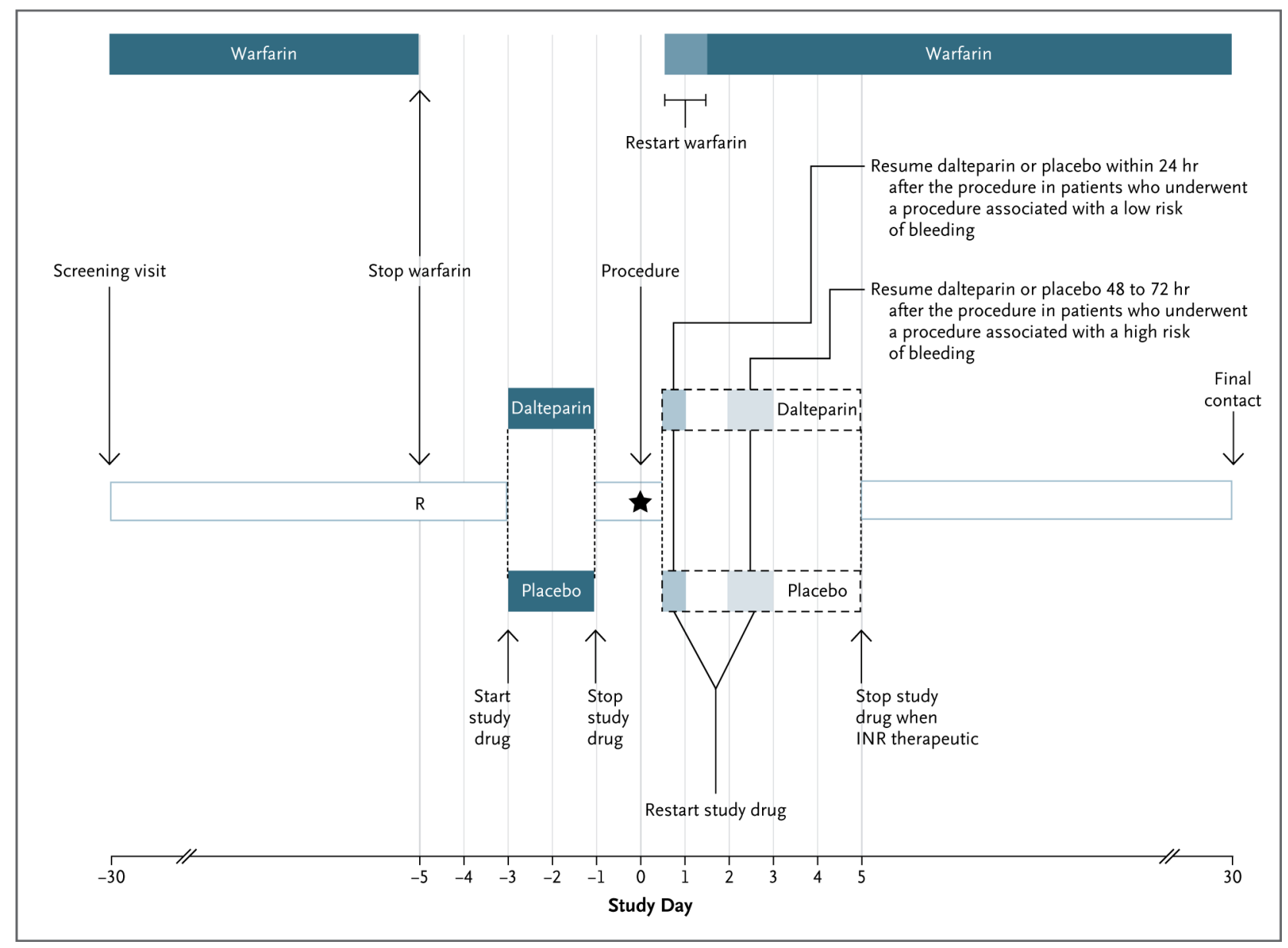

Figure 1. BRIDGE Study Design

Screening visits occurred between 30 days and 5 days before the planned procedure, and randomization $(\mathrm{R})$ occurred 5 days before the procedure. Warfarin treatment was discontinued 5 days before the procedure, and administration of the study drug was initiated 3 days before the procedure. It was recommended that the international normalized ratio (INR) be measured 1 day before the procedure; if the INR was greater than 1.8 , oral vitamin $\mathrm{K}$ (1.0 to $2.5 \mathrm{mg}$ ) was recommended; if the INR was 1.5 to 1.8 , oral vitamin $\mathrm{K}$ was optional. If the procedure or surgery was delayed up to 3 days, administration of the study drug was continued until 24 hours before the procedure. Warfarin treatment was restarted on the evening of or the day after the procedure, and the study drug was restarted 12 to 24 hours after a minor (or low-bleeding-risk) procedure and 48 to 72 hours after a major (or highbleeding-risk) procedure. Administration of the study drug was continued after the procedure until the INR was 2.0 or higher on one occasion. The final patient follow-up occurred 30 days after the procedure. LMWH denotes low-molecular-weight heparin. 


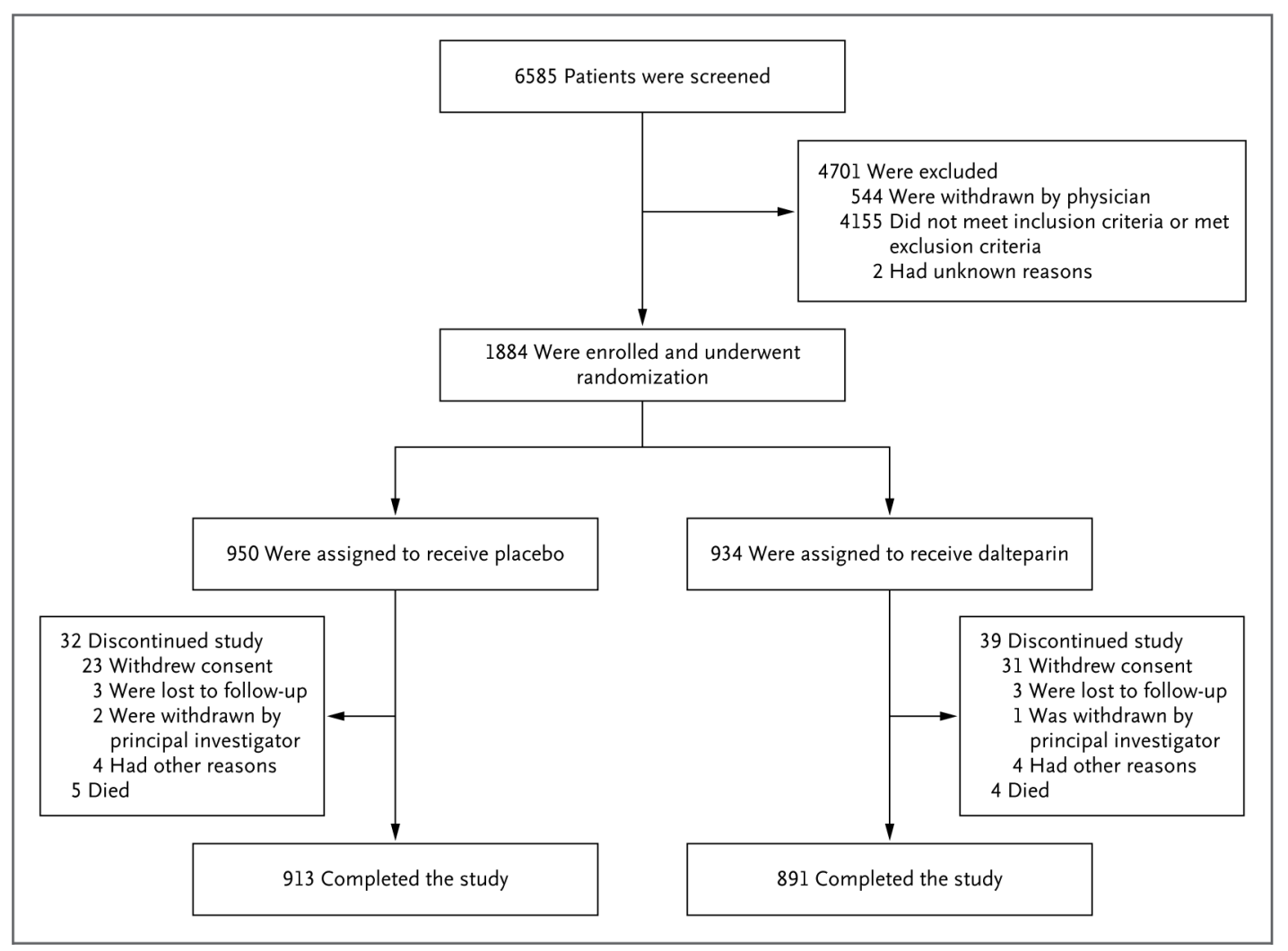

Figure 2.

Screening, Randomization, and Follow-up. 
Table 1

Baseline Characteristics of the Patients. *

\begin{tabular}{|c|c|c|}
\hline Characteristic & No Bridging $(\mathrm{N}=950)$ & Bridging $(N=934)$ \\
\hline Age $-\mathrm{yr}$ & $71.8 \pm 8.74$ & $71.6 \pm 8.88$ \\
\hline Male sex — no. $(\%)$ & $696(73.3)$ & $686(73.4)$ \\
\hline \multicolumn{3}{|l|}{ Race - no. $(\%)^{\dagger}$} \\
\hline White & $860(90.5)$ & $849(90.9)$ \\
\hline Nonwhite & $88(9.3)$ & $82(8.8)$ \\
\hline Unknown & $2(0.2)$ & $3(0.3)$ \\
\hline Weight $-\mathrm{kg}$ & $96.2 \pm 24.87$ & $95.4 \pm 23.50$ \\
\hline \multicolumn{3}{|l|}{ CHADS $_{2}$ score $^{* t}$} \\
\hline Mean & $2.3 \pm 1.03$ & $2.4 \pm 1.07$ \\
\hline \multicolumn{3}{|l|}{ Distribution — no. $(\%)$} \\
\hline 0 & $1(0.1)$ & $1(0.1)$ \\
\hline 1 & $216(22.7)$ & $212(22.7)$ \\
\hline 2 & $382(40.2)$ & $351(37.6)$ \\
\hline 3 & $229(24.1)$ & $232(24.8)$ \\
\hline 4 & $96(10.1)$ & $106(11.3)$ \\
\hline 5 & $23(2.4)$ & $27(2.9)$ \\
\hline 6 & $3(0.3)$ & $5(0.5)$ \\
\hline CHF or left ventricular dysfunction - no. (\%) & $289(30.4)$ & $310(33.2)$ \\
\hline Hypertension — no. (\%) & $833(87.7)$ & $806(86.3)$ \\
\hline Diabetes mellitus — no. (\%) & $390(41.1)$ & $382(40.9)$ \\
\hline Stroke - no. $(\%)$ & $79(8.3)$ & 99 (10.6) \\
\hline Transient ischemic attack - no. (\%) & $79(8.3)$ & $77(8.2)$ \\
\hline Mitral valve disease — no. $(\%)$ & $165(17.4)$ & $142(15.2)$ \\
\hline Stenosis & $19(2.0)$ & $10(1.1)$ \\
\hline Regurgitation & $142(14.9)$ & $133(14.2)$ \\
\hline Prolapse & $13(1.4)$ & $5(0.5)$ \\
\hline Myocardial infarction — no. (\%) & $138(14.5)$ & $155(16.6)$ \\
\hline Renal disease — no. $(\%)$ & $108(11.4)$ & $92(9.9)$ \\
\hline Solid malignant disease - no. (\%) & $68(7.2)$ & $52(5.6)$ \\
\hline \multicolumn{3}{|l|}{ Laboratory values } \\
\hline Hemoglobin $-\mathrm{g} / \mathrm{dl}$ & $13.8 \pm 1.67$ & $13.8 \pm 1.62$ \\
\hline Platelet count — thrombocytes $/ \mathrm{mm}^{3}$ & $209,300 \pm 592,900$ & $209,200 \pm 580,500$ \\
\hline INR & $2.4 \pm 0.57$ & $2.4 \pm 0.57$ \\
\hline Serum creatinine $-\mathrm{mg} / \mathrm{dl}$ & $1.1 \pm 0.32$ & $1.1 \pm 0.32$ \\
\hline Creatinine clearance $-\mathrm{ml} / \mathrm{min}$ & $88.1 \pm 39.50$ & $87.6 \pm 40.14$ \\
\hline Medication use - no. (\%) & & \\
\hline
\end{tabular}

N Engl J Med. Author manuscript; available in PMC 2016 July 04. 


\begin{tabular}{|lcc|}
\hline Characteristic & No Bridging $(\mathbf{N}=\mathbf{9 5 0})$ & Bridging $(\mathbf{N}=\mathbf{9 3 4})$ \\
\hline Aspirin & $324(34.1)$ & $329(35.2)$ \\
\hline Clopidogrel & $30(3.2)$ & $21(2.2)$ \\
\hline Nonsteroidal antiinflammatory drug & $34(3.6)$ & $25(2.7)$ \\
\hline COX-2 inhibitor & $8(0.8)$ & $13(1.4)$ \\
\hline
\end{tabular}

Plus-minus values are means $\pm \mathrm{SD}$. There were no significant differences between the groups $(\mathrm{P}<0.05)$. CHF denotes congestive heart failure, COX-2 cyclooxygenase type 2 , and INR international normalized ratio.

${ }^{\dagger}$ Race was self-reported. The patients for whom data were unknown are those who chose not to provide information.

${ }^{t} \mathrm{CHADS}_{2}$ is a score used to estimate the risk of stroke in patients with atrial fibrillation. The score ranges from 1 to $6 ; 1$ point each is assigned for congestive heart failure, hypertension, age of 75 years or older, and diabetes mellitus, and 2 points are assigned for stroke or transient ischemic attack. 


\section{Table 2}

Perioperative Anticoagulant Management.

\begin{tabular}{|c|c|c|c|}
\hline Variable & No Bridging $(\mathrm{N}=950)$ & Bridging $(\mathrm{N}=934)$ & P Value \\
\hline \multicolumn{4}{|l|}{ Warfarin treatment } \\
\hline Preprocedure time not taking warfarin & & & 0.28 \\
\hline No. of patients with data & 872 & 839 & \\
\hline Mean — days & $5.2 \pm 1.4$ & $5.3 \pm 1.8$ & \\
\hline Time to first postprocedure warfarin dose & & & 0.40 \\
\hline No. of patients with data & 735 & 696 & \\
\hline Mean — days & $1.5 \pm 1.3$ & $1.4 \pm 1.0$ & \\
\hline \multicolumn{4}{|l|}{ Low-molecular-weight heparin or placebo } \\
\hline Preprocedure dose & & & 0.61 \\
\hline No. of patients with data & 796 & 768 & \\
\hline Mean no. of doses & $5.0 \pm 0.7$ & $5.0 \pm 1.4$ & \\
\hline $\begin{array}{l}\text { Patients in whom the last dose was taken on the morning of the day before the } \\
\text { procedure - no./total no. (\%) }\end{array}$ & 778/796 (97.7) & $734 / 768(95.6)$ & 0.02 \\
\hline \multicolumn{4}{|l|}{ Time to first postprocedure dose } \\
\hline Major surgery or procedure (high bleeding risk) & & & 0.74 \\
\hline No. of patients with data & 235 & 223 & \\
\hline Mean - hr & $53.3 \pm 31.6$ & $51.3 \pm 27.9$ & \\
\hline Minor surgery or procedure (low bleeding risk) & & & 0.74 \\
\hline No. of patients with data & 526 & 497 & \\
\hline Mean - hr & $21.1 \pm 2.3$ & $21.0 \pm 2.4$ & \\
\hline Postprocedure dose & & & 0.47 \\
\hline No. of patients with data & 764 & 721 & \\
\hline Mean no. of doses & $15.7 \pm 7.4$ & $16.1 \pm 8.4$ & \\
\hline Aspirin treatment - no./total no. (\%) & & & 0.53 \\
\hline Interruption $\geq 7$ days before procedure & $92 / 324(28.4)$ & $92 / 329(28.0)$ & \\
\hline Interruption $<7$ days before procedure & $41 / 324(12.7)$ & $33 / 329(10.0)$ & \\
\hline No interruption & $191 / 324(59.0)$ & $204 / 329(62.0)$ & \\
\hline
\end{tabular}


Table 3

Study Outcomes.

\begin{tabular}{|lccc|}
\hline Outcome & $\begin{array}{l}\text { No Bridging }(\mathbf{N}=918) \\
\text { number of patients (percent) }\end{array}$ & Bridging (N = 895) & P Value \\
\hline Primary & & & \\
\hline Arterial thromboembolism & $4(0.4)$ & $3(0.3)$ & $0.01^{*}, 0.73^{\dagger}$ \\
\hline Stroke & $2(0.2)$ & $3(0.3)$ & \\
\hline Transient ischemic attack & $2(0.2)$ & 0 & \\
\hline Systemic embolism & 0 & 0 & $0.005^{\dagger}$ \\
\hline Major bleeding & $12(1.3)$ & $29(3.2)$ & \\
\hline Secondary & & $4(0.4)$ & $0.88^{\dagger}$ \\
\hline Death & $5(0.5)$ & $14(1.6)$ & $0.10^{\dagger}$ \\
\hline Myocardial infarction & $7(0.8)$ & $1(0.1)$ & $0.25^{\dagger}$ \\
\hline Deep-vein thrombosis & 0 & $1(0.1)$ & $0.25^{\dagger}$ \\
\hline $\begin{array}{l}\text { Pulmonary embolism } \\
\text { Minor bleeding }\end{array}$ & $110(12.0)$ & $0.001^{\dagger}$ \\
\hline
\end{tabular}

* $\mathrm{P}$ value for noninferiority.

${ }^{\dagger} \mathrm{P}$ value for superiority. 